\title{
Spectral analysis of PG 1034+001, the exciting star of Hewett 1
}

\author{
Marin Mahsereci ${ }^{1}$, Ellen Ringat ${ }^{1}$, Thomas Rauch ${ }^{1}$, Klaus Werner ${ }^{1}$ \\ and Jeffrey W. Kruk ${ }^{2}$ \\ ${ }^{1}$ Institute for Astronomy and Astrophysics, Kepler Center for Astro and Particle Physics, \\ Eberhard Karls University, Sand 1, 72076 Tübingen, Germany \\ email: maren.mahsereci@astro.uni-tuebingen.de \\ ${ }^{2}$ NASA Goddard Space Flight Center, Greenbelt, MD 20771, U.S.A.
}

\begin{abstract}
PG 1034+001 is an extremely hot, helium-rich DO-type star that excites the planetary nebula Hewett 1 and large parts of the surrounding interstellar medium. We present preliminary results of an ongoing spectral analysis by means of non-LTE model atmospheres that consider most elements from hydrogen to nickel. This analysis is based on high-resolution ultraviolet (FUSE, IUE) and optical (VLT/UVES, Keck) data. The results are compared with those of PG 1034+001's spectroscopic twin, the DO star PG 0038+199.
\end{abstract}

Keywords. stars: abundances, stars: AGB and post-AGB, stars: atmospheres, stars: evolution, stars: individual (PG 1034+001, PG 0038+199), planetary nebulae: individual (Hewett 1)

\section{Introduction}

PG 1034+001 is an extremely hot, helium-rich DO-type star that was discovered in the Palomar Green Survey. It is surrounded by the planetary nebula Hewett 1 (Hewett et al. 2003) and at least one big emission shell (Rauch et al. 2004). PG 1034+001 is located at the hot end of the white dwarf cooling sequence and, thus, it is well suited to provide constraints on stellar evolutionary theory studies. First LTE and non-LTE stellar model-atmosphere calculations were performed by Wesemael et al. $\left(1985, T_{\text {eff }}=\right.$ $80000 \mathrm{~K}, \log g \geqslant 6)$ and Werner et al. $\left(1995, T_{\mathrm{eff}}=100000 \mathrm{~K}, \log g=7.5\right)$. Improved model atoms and increased computational power now allow for a precise reanalysis.

PG 0038+199 was also discovered in the Palomar Green Survey. Like PG 1034+001, it is an extremely hot, DO-type star. The first non-LTE model-atmosphere analysis was performed by Dreizler \& Werner $\left(1996, T_{\text {eff }}=115000 \mathrm{~K}, \log g=7.5\right)$.

\section{Observations and Analysis}

The main data source for the analysis of PG 1034+001 and PG 0038+199 is FUSE (Far Ultraviolet Spectroscopic Explorer, ProgID: P104). FUSE operated in the far ultraviolet range from about $905-1187 \AA$ with a resolution of $\approx 0.06 \AA$. This region exhibits many iron-group lines that are, due to the high resolution, well resolved. UV spectra from IUE (International Ultraviolet Explorer) and optical spectra are used for checks.

TMAP (Tübingen NLTE Model-Atmosphere Package†) is a tool to compute non-LTE model atmospheres. It assumes that atmospheres are plane-parallel and in hydrostatic and radiative equilibrium. TMAP can consider elements from hydrogen to nickel with about 1500 NLTE levels. Ready-to-use model atoms are provided by TMAD (Tübingen

$\dagger$ http://astro.uni-tuebingen.de/ TMAP/TMAP.html 
Table 1. Preliminary results of $T_{\text {eff }}(\mathrm{kK}), \log g\left(\mathrm{~cm} / \mathrm{sec}^{2}\right)$, and abundances (mass fraction) of PG 1034+001 and PG 0038+199. The errors are about $15 \%$ for $T_{\text {eff }}, 0.3$ for $\log g$, and 0.3 for abundances.

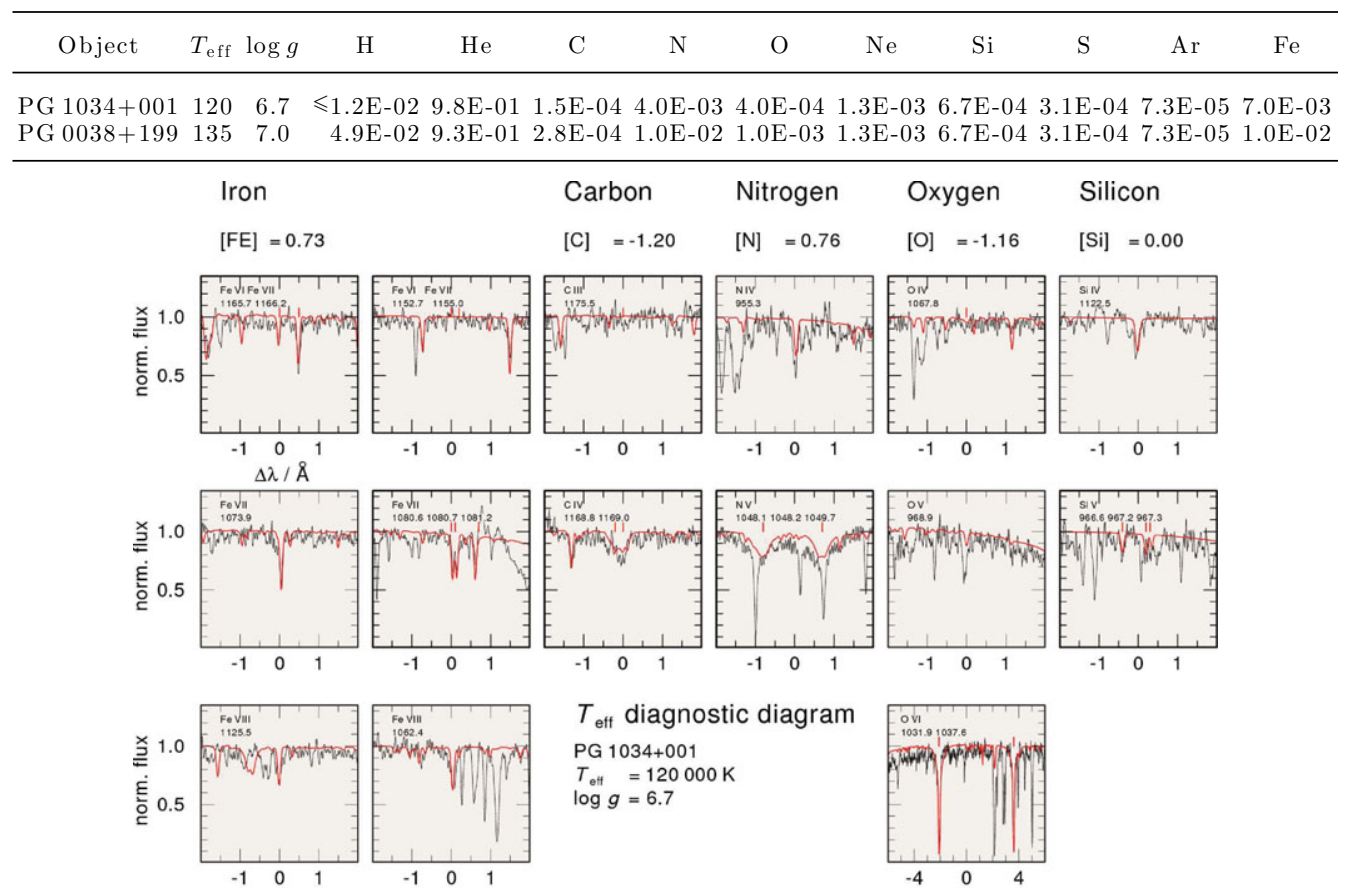

Figure 1. Diagram to determine $T_{\text {eff }}$ of PG $1034+001$. The model accounts for opacities of the elements $\mathrm{H}, \mathrm{He}, \mathrm{C}, \mathrm{N}, \mathrm{O}$, and $\mathrm{Ca}-\mathrm{Ni}$. Si is included in a line-formation procedure. The abundances $[\mathrm{X}]$ are given as logarithm relative to the solar abundance.

Model Atom Database $\ddagger$ ). Due to the many lines and levels of iron-group elements their cross sections are computed with the program $\operatorname{IrOnIc}$ using a statistical method and then included into the TMAP calculations. We use the ionization balance of several elements to determine $T_{\text {eff }}$. Especially iron is very sensitive to temperature changes and exhibits three ionization stages (VI-VIII, Fig. 1). The abundances (Tab. 1) are in agreement with expectations of evolutionary models (Werner \& Herwig 2006). The inclusion of iron-group opacities and the availability of high resolution FUSE data lead to the revised values of $T_{\text {eff }}$ and $\log g$. The change in $T_{\text {eff }}$ is comparable to the uncertainties, but the decrease in $\log g$ is significant.

\section{Acknowledgements}

TR is supported by the German Aerospace Center (DLR) under grant 05 OR 0806, ER by the Federal Ministry of Education and Research (BMBF) under grant 05A11VTB.

\section{References}

Dreizler S. \& Werner K. 1996, A\& A, 314, 217

Hewett, P. C., Irwin, M. J., Skillman, E. D., et al. 2003, ApJ, 599, L37

Rauch T., Kerber F., \& Pauli E.-M. 2004, A\&SA, 417, 647

Werner, K. \& Herwig, F. 2006, PASP, 118, 183

Werner K., Dreizler S., \& Wolff B. 1995, A\&A, 298, 567

Wesemael F., Green R. F., \& Liebert J. 1985, ApJ, 307, 659 